\title{
Spin dynamics near the quantum critical point of heavy fermions
}

\author{
C. Pépin ${ }^{\mathrm{a}, *}$, M. Lavagna ${ }^{\mathrm{b}}$

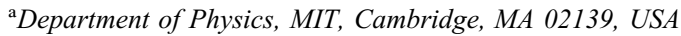 \\ ${ }^{\mathrm{b}}$ Commissariat à l'Energie Atomique, DRFMC/SPSMS, 38054 Grenoble Cedex 9, France
}

\begin{abstract}
The dynamical spin susceptibility is studied in the magnetically disordered phase of heavy-fermion systems near the antiferromagnetic quantum phase transition. In the framework of the $S=\frac{1}{2}$ Kondo lattice model, we introduce a perturbative expansion treating the spin and Kondo-like degrees of freedom on an equal footing. The general expression of the dynamical spin susceptibility that we derive presents a two-component behaviour: a quasielastic peak as in a Fermi liquid theory, and a strongly $\boldsymbol{q}$-dependent inelastic peak typical of a non-Fermi liquid behaviour. Very strikingly, the position of the inelastic peak is found to be pushed to zero at the antiferromagnetic transition with a vanishing relaxation rate. The comparison has been quantitatively made with inelastic neutron scattering (INS) experiments performed in $\mathrm{CeCu}_{6}$ and $\mathrm{Ce}_{1-x} \mathrm{La}_{x} \mathrm{Ru}_{2} \mathrm{Si}_{2}$. The excellent agreement that we have found gives strong support to a two-band model with new prospects for the study of the quantum critical phenomena in the vicinity of the magnetic phase transition. (C) 1999 Published by Elsevier Science B.V. All rights reserved.
\end{abstract}

Keywords: Kondo lattice model; Dynamical spin susceptibility; Heavy fermion

One of the most striking properties of heavy-fermion compounds discovered these last years is the existence of a quantum phase transition driven by composition change $\left(\right.$ at $x_{\mathrm{C}}=0.1$ in $\mathrm{CeCu}_{6-x} \mathrm{Au}_{x}$ and $x_{\mathrm{C}}=0.08$ in $\mathrm{Ce}_{1-x} \mathrm{La}_{x} \mathrm{Ru}_{2} \mathrm{Si}_{2}$ ), pressure or magnetic field. Important insight is provided by the evolution of the low-temperature neutron cross section measured by inelastic neutron scattering (INS) experiments when getting closer to the magnetic instability. The experiments performed in pure compounds $\mathrm{CeCu}_{6}$ and $\mathrm{CeRu}_{2} \mathrm{Si}_{2}$ by Regnault et al. $[1,17,18]$ and Aeppli et al. [2] have shown the presence of two distinct contributions to the dynamic magnetic structure factor: a $\boldsymbol{q}$-independent quasielastic component, and a strongly $\boldsymbol{q}$-dependent inelastic peak with a maximum at the value $\omega_{\max }$ of the frequency. The former corresponds to localized excitations of Kondotype while the latter peaked at some wavevector $\boldsymbol{Q}$ is

* Corresponding author. believed to be associated with intersite magnetic correlations due to RKKY interactions. Both the position and the width of the inelastic peak vanish when getting near the magnetic instability.

Any theory aimed to describe the quantum critical phenomena in heavy-fermion compounds should account for the so-quoted behaviour of the dynamical spin susceptibility. We start from the Kondo lattice model which is believed to describe the physics of these systems. Most of the theories developed so far [3,4,8,9] agree with the existence of a hybridization gap which splits the Abrikosov-Suhl or Kondo resonance formed at the Fermi level. The role of the interband transitions has been outlined for long in order to explain the inelastic component of the dynamical spin susceptibility. For instance, the theories based on a $1 / N$ expansion [3-7] (where $N$ is simultaneously the degeneracy of the conduction electrons and of the spin channels) predict a maximum of $\chi^{\prime \prime}\left(k_{F}, \omega\right) / \omega$ at $\omega_{\max }$ of the order of the indirect hybridization gap [10]. However, the $1 / N$ expansion 
theories present serious drawbacks: (i) the spin fluctuation effects are automatically ruled out since the RKKY interactions only occur at the following order in $1 / N^{2}$ [11], (ii) they then fail to describe any magnetic instability and hence the quantum critical phenomena mentioned above and (iii) the predictions for $\omega_{\max }$ and the associated relaxation rate cannot account for the experimental observations near the magnetic instability. An improvement brought by Doniach [12] and followed by other authors [13] considers the $1 / N^{2}$ corrections in an instantaneous approximation: it gives back the ladder diagram contribution to the dynamical spin susceptibility and then accounts for the spin fluctuation effects. But still the predictions for the frequency dependence of the dynamic magnetic structure factor presents a gap of the order of the hybridization gap whatever the value of the interaction is. On the other hand, in front of the difficulties encountered when starting from microscopic descriptions, various phenomenological models (as the duality model of Kuramoto and Miyake [14] or that of Bernhoeft and Lonzarich [15]) have been introduced to describe both the spin fluctuation and the itinerant electron aspects with some successful predictions as the weak antiferromagnetism of these systems.

In this work, we have developed a systematic approach to the Kondo lattice model for $S=\frac{1}{2}(N=2)$ in which the Kondo-like and the spin degrees of freedom are treated on an equal footing (see our longer paper [16] for further details). The saddle-point results and the Gaussian fluctuations in the charge channel are consistent with the standard $1 / N$ theories with the existence of two bands corresponding to the formation of a Kondo resonance split by a hybridization gap. For $n_{\mathrm{c}}<1$, the chemical potential is located below the upper edge of the $\alpha$ band and the system is metallic. The Gaussian fluctuations in the spin channel restore the spin fluctuation effects which were missing in the $1 / N$ expansion. The general expression of the dynamical spin susceptibility $\chi_{\mathrm{ff}}(\boldsymbol{q}, \omega)$ shows two contributions corresponding to intraand interband transitions. Fig. 1 reports the contiuum of intraband and interband particle-hole pair excitations. Far from the antiferromagnetic vector $\boldsymbol{Q}, \chi_{\mathrm{ff}}(\boldsymbol{q}, \omega)$ is dominated by the intraband contribution. In this case, the frequency dependence of the dynamical spin susceptibility is Lorentzian in the low-frequency limit as in a standard Fermi liquid theory. On the other hand, we have shown how at the antiferromagnetic wave vector, $\chi_{\mathrm{ff}}(\boldsymbol{Q}, \omega)$ is driven by the interband transitions giving rise to an inelastic peak. In a very striking way, the value $\omega_{\max }$ of the maximum of $\chi_{\text {inter }}^{\prime \prime}(\boldsymbol{Q}, \omega) / \omega$ is pushed to zero at the antiferromagnetic phase transition and the inelastic mode becomes soft with vanishing relaxation rate.

We believe that the two contibutions that we have found respectively correspond to the quasielastic and inelastic peaks observed by INS. The role of the interband transitions had already been pointed out in pre-

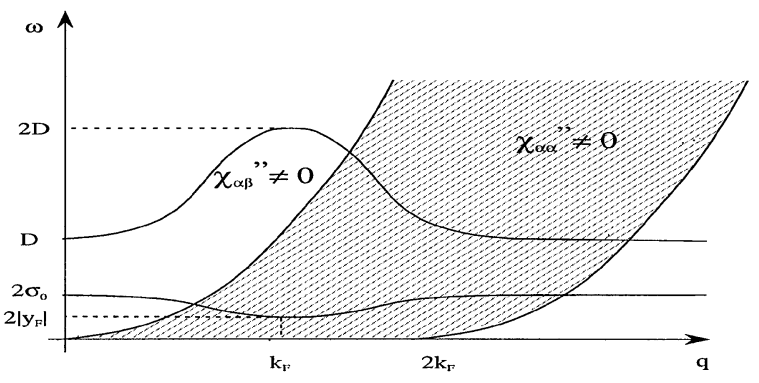

Fig. 1. Continuum of the intra- and interband electron-hole pair excitations $\chi_{\alpha \alpha}^{\prime \prime}(q, \omega) \neq 0$ and $\chi_{\alpha \beta}^{\prime \prime}(q, \omega) \neq 0$. Note the presence of a gap in the interband transitions equal to the indirect gap at $q=k_{F}$, and to the direct gap at $q=0$.

vious works [10]. However, while the previous studies conclude the presence of an inelastic peak at finite value of the frequency related to the hybridization gap whatever the interaction $J$ is, the originality of our approach is to predict a renormalization of both $\omega_{\max }$ and the relaxation rate due to spin fluctuation effects. The comparison has been quantitatively made with INS experiments performed in $\mathrm{CeCu}_{6}$ and $\mathrm{Ce}_{1-x} \mathrm{La}_{x} \mathrm{Ru}_{2} \mathrm{Si}_{2}$. The quasielastic peak is typical of a Fermi liquid while the other mode breaks the Fermi liquid description. For this reason and because of the very good agreement of our results with the INS experiments, our approach offers news prospects for the study of the quantum critical phenomena in the vicinity of the antiferromagnetic phase transition.

\section{References}

[1] L.P. Regnault, W.A.C. Erkelens, J. Rossat-Mignod, P. Lejay, J. Flouquet, Phys. Rev. B 38 (1988) 4481.

[2] G. Aeppli, H. Yoshizawa, Y. Endoh, E. Bucher, J. Hufnagl, Phys. Rev. Lett. 57 (1986) 122.

[3] A.J. Millis, P.A. Lee, Phys. Rev. B 35 (1987) 3394.

[4] A. Auerbach, K. Levin, Phys. Rev. Lett. 57 (1986) 877.

[5] M. Lavagna, A.J. Millis, P.A. Lee, Phys. Rev. Lett. B 58 (1987) 266.

[6] P. Coleman, Phys. Rev. B 29 (1984) 3035.

[7] N. Read, D.N. Newns, J. Phys. C 16 (1983) 3273.

[8] C. Lacroix, M. Cyrot, Phys. Rev. B 20 (1979) 1969.

[9] T.M. Rice, K. Ueda, Phys. Rev. Lett. B 55 (1985) 995.

[10] A. Auerbach, Ju H. Kim, K. Levin, M.R. Norman, Phys. Rev. Lett. 60 (1988) 623.

[11] A. Houghton, N. Read, H. Won, Phys. Rev. B 37 (1988) 3782.

[12] S. Doniach, Phys. Rev. B 35 (1987) 1814.

[13] C. Lacroix, J. Magn. Magn. Mater. 100 (1991) 90.

[14] Y. Kuramoto, K. Miyake, J. Phys. Soc. Japan 59 (1990) 2831.

[15] N.R. Bernhoeft, G.G. Lonzarich, J. Phys. Cond. Mater. 7 (1995) 7325.

[16] C. Pépin, M, Lavagna, Cond-Mat Preprint 9803255.

[17] S. Raymond, L.P. Regnault, S. Kambe, J.M. Mignod, P. Lejay, J. Flouquet, J. Low Temp. Phys. 109 (1997) 205.

[18] S. Raymond, L.P. Regnault, S. Kambe, J.M. Mignod, P. Lejay, J. Flouquet, in these Proceedings (SCES '98), Physica B 259-261 (1999). 